\title{
Are Few-Mode Fibres a Practical Solution to the Capacity Crunch?
}

\author{
Andrew Ellis*, Nick Doran \\ Aston Institute of Photonic Technologies, Aston University, Birmingham, UK \\ Tel: (44) 121204 3075, e-mail: andrew.ellis@aston.ac.uk
}

\begin{abstract}
In this paper, we compare the nonlinear Shannon capacity of few-mode fibre systems operating with spatialtemporal digital signal processing to the nonlinear Shannon capacity of single-mode fibre systems operating with spectral-temporal digital signal processing. Combining these results with estimates of digital signal processing complexity for each option offers valuable insights to system designers.
\end{abstract}

Keywords: nonlinear Shannon capacity, few-mode fibre, spatial multiplexing.

\section{INTRODUCTION}

Widespread acceptance of the fundamental fibre capacity limits has recently been crystallised through various analytical calculations of the so called nonlinear Shannon limit, based on single channel calculations, cross phase modulation for WDM systems [1] and four-wave mixing for OFDM super-channels and channels with arbitrary spectral profiles [2]. Provided their underlying assumptions remain valid, these analytical theories accurately predict the performance of numerically simulated and experimentally demonstrated systems, further increasing confidence in their predictions. Indeed recent experimental results, either plotted as a fraction of the theoretical limit for a given experiment [3], or in terms of raw capacity (Fig. 1) show clear signs of saturation. Figure 1, which compares experimental results and commercial systems in access and core networks, suggests that the capacity crunch will occur in the middle of the next decade. This motivates a wide variety of research activities including spatial multiplexing using few-mode and multi-core fibre (allowing $1 \mathrm{Pbit} / \mathrm{s}$ to be transported within a single fibre cladding [4]), renewed interest in optical regeneration, but for wavelength division multiplexed (WDM) [5] and QAM formatted signals [6], optical [7] and electrical [8] compensation of nonlinearity and ultralow noise links using phase sensitive amplification [9].

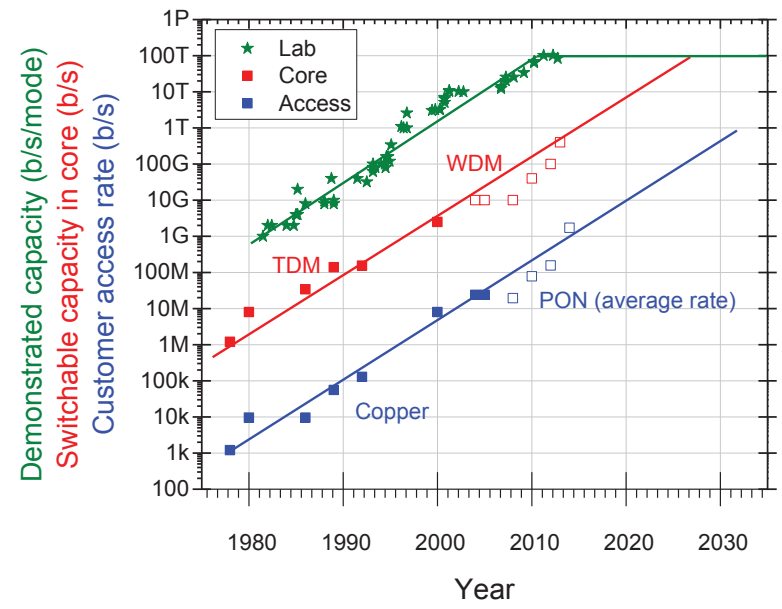

Figure 1. Capacity evolution of communication networks showing: Blue squares, data services offered to residential customers using copper access technologies (solid squares) or passive optical networks (open squares, statistically multiplexed); Red circles, switched capacity in core network including time division multiplexed systems such as PDH and $S D H$ (solid circles) and wavelength routed systems (open circles); Green stars, reported laboratory results taken from ECOC and OFC post deadline sessions and other notable papers.

In this paper, we consider the nonlinear Shannon limits of various potential fibre infrastructures and estimate the potential energy consumptions of each option. We show that few mode fibres should support at least 4 LP modes in order to ensure that their capacity limits exceed the limit of a conventional SMF employing advanced multi-channel digital back propagation (DBP).

\section{METHOD}

In common with previous calculations for single mode fibre, we consider the nonlinear interaction from the Kerr nonlinearity in the form of four-wave mixing (FWM). All phase matched combinations of wavelengths and modes will contribute to the nonlinear noise generated in the fibre and assuming that all contributions are independent then the nonlinear Shannon limit for an $M$ mode fibre with $N_{s}$ spans is given by; 


$$
\left.\frac{C}{B}\right|_{M}=\sum_{m=1}^{M} \log _{2}\left(1+\frac{P_{m}}{N_{s} P_{a s e}+N_{s} \sum_{i, j, k} \eta_{i j k m}(B) P_{i} P_{j} P_{k}}\right)
$$

for an $M$ mode fibre, where $C$ is the information carried (b/s) in a bandwidth $B, P_{m}$ is the power spectral density of the signal in mode $m, P_{\text {ase }}$ the noise power spectral density per mode and $\eta_{i j k m}$ is a nonlinear efficiency obtained by a double integral in frequency for each possible combination of modes. One may immediately determine the minimum benefit of spatial multiplexing through the highly pessimistic approximation that the nonlinear interaction between all modes have equal efficiencies (identical effective areas, zero DMD), resulting in an $M$ fold decrease in the optimum nonlinear signal to noise ratio (SNR). Despite this increase in noise the overall capacity increases monotonically with the number of modes, although beyond around 10 modes, the increase is small [3]. More accurately the nonlinear efficiency may be calculated by integrating the quasi phase matched Maker fringes [2] over the signal bandwidth taking into account any phase matching offsets induced by differential mode delays [10]. In this case $\eta_{i j k m}$ is given by [11]

$$
\begin{aligned}
\eta_{i j k m}(B) & =\frac{\xi_{i j k n}}{A_{i j k n}^{2}} \frac{\omega_{0}^{2} n_{2}^{2}}{c^{2} \pi \alpha\left|\beta_{2, n}\right|} \cdot\left\{\ln \left(\frac{B^{2}+2 B \Delta f_{i j k n}}{2 \cdot f_{w}^{2}}\right)+\operatorname{s} \cdot \ln \left(s \frac{B^{2}-2 B \Delta f_{i j k n}}{2 \cdot f_{w}^{2}}\right)\right\} \\
s & =\operatorname{Sign}(B-2 \Delta f) \quad f_{w}=\sqrt{\alpha / 4 \pi^{2} \beta}
\end{aligned}
$$

where we have adopted the usual meaning for $\omega, \alpha, \beta, n_{2}$ and $c . N_{s}$ represents the number of spans, $f_{w}$ a characteristic frequency defining the width of the FWM efficiency curve, $\Delta f_{i j k m}$ the frequency detuning for maximum phase matching, $A_{i j k m}$ the effective area of the inter mode interaction and $\xi_{i j k m}$ a parameter accounting for the reduction in nonlinear efficiency associated with degenerate modes[12] (a value of $2 / 3$ is assumed here). Note that $\eta_{i j k m}$ is zero for large fraction of the potential inter mode interactions and vanishingly small if $\Delta f_{i j k m}$ » $B$, consequently, only a small fraction of the inter mode interactions contribute to the nonlinear noise in eq. (1). Following this more accurate calculation, we find that a significantly larger number of modes may be employed, provided the linear impairments may be compensated with acceptable accuracy.

For a system employing DBP or transmitter based optical phase conjugation (OPC), the inter signal noise terms detected within the bandwidth of the DBP system are compensated, and may simply be subtracted from the nonlinear noise. However, eventually, the system becomes limited by parametric noise amplification [13], and for accurate calculations this term should be added to the denominator of eq. (1). Assuming equal signal power levels throughout to simplify the expression, the nonlinear Shannon limit is then:

$$
\left.\frac{C}{B}\right|_{M}=\sum_{m=1}^{M} \log _{2}\left(1+\frac{P_{S}}{N_{s} P_{a s e}+\sum_{i, j, k}\left[N_{s}\left\{\eta_{i j k m}\left(B_{W D M}\right)-\eta_{i j k m}\left(B_{D B P}\right)\right\} P_{S}^{3}+\sum_{n=1}^{N_{s}} n \eta_{i j k m}\left(B_{W D M}\right) P_{a s e} P_{S}^{2}\right]}\right)
$$

Recently, calculations of the equivalent Shannon capacity of optically regenerated systems have been performed; showing that with practically achievable regenerator transfer functions for QAM formatted data, the equivalent Shannon capacity of the regenerated system lies somewhere between the nonlinear Shannon capacity of the link without regenerators and that of a single span system. These calculations take into account error accumulation between regenerators, and incomplete noise squeezing [14]. In this paper, we assume ideal transfer functions for simplicity (noiseless regenerator outputs), and account for the error accumulation by imposing an nonlinear SNR penalty of $\varepsilon$ given by;

$$
\varepsilon=\frac{q-1}{3 . s n r \cdot \log _{2}(q)} W\left(\frac{3 \cdot s n r \cdot \log _{2}(q)}{q-1} N_{r}^{2} m^{\frac{3 \cdot s n r .}{\ln (2)(q-1)}}\right)
$$

where $\mathrm{W}$ is the Lambert $\mathrm{W}$ function, such that $q-Q A M$ regenerator based systems with $N_{r}$ regenerators require a SNR of $\varepsilon . s n r$ to provide the same performance as a single span system with a SNR of snr.

\section{RESULTS}

Figure 2a shows the optimum nonlinear Shannon capacity of various $6,000 \mathrm{~km}$ systems with total bandwidths of $5 \mathrm{THz}, 80 \mathrm{~km}$ amplifier spacing $(4.5 \mathrm{~dB}$ noise figures) and $50 \mathrm{GHz}$ channel spacing considering few mode fibres, and single mode fibres employing regenerators or DBP. Conventional single mode fibre gives a capacity 
limit of $5.6 \mathrm{~b} / \mathrm{s} / \mathrm{Hz}$ (solid green), whilst 2 and 4-mode fibres with high DMD offer limits of $15 \mathrm{~b} / \mathrm{s} / \mathrm{Hz}$ (purple) and 31 (black) respectively reflecting small penalties with respect to the expected 3-fold and 6-fold enhancements from the additional modes. However, the signal processing complexities of these fibres is immense, due to the large DMD $(2.13 \mathrm{~ns} / \mathrm{km}$ for the 4 -mode fibre $)$ and the MIMO processing. To address this, the DMD may be reduced through appropriate fibre optimisation at the expense of enhanced inter-mode velocity matching resulting in larger non-linear interactions (light blue and dark purple). With low DMD fibres (170 ps/nm for the 4 mode fibre), we can observe that the loss of capacity with respect to equivalent SNF can readily exceed the capacity of one complete SMF with the 2-mode fibre only offering 2.3 times the capacity of SMF at the expense of a threefold increase in the MIMO ports, whilst the 4-mode fibre only offers 4.5 times the capacity of a SMF.
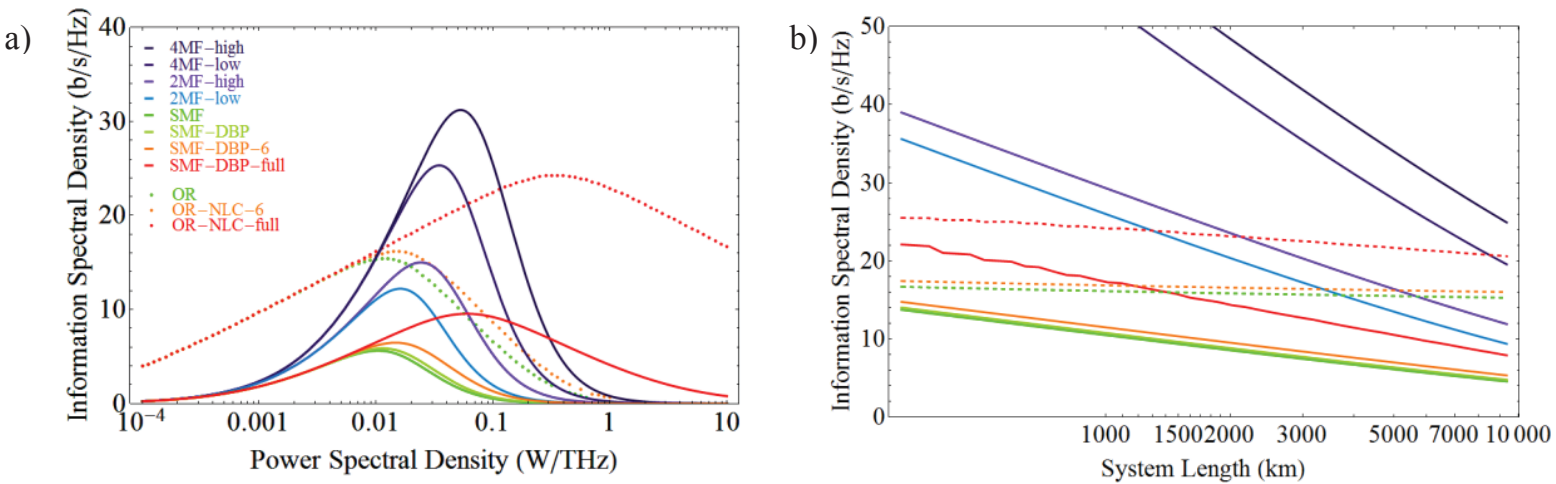

Figure 2. Information spectral density for systems with $5 \mathrm{THz}$ bandwidth, $100 \mathrm{~km}$ amplifier spacing (4.5 dB noise figures), 50 GHz channel spacing, $0.2 \mathrm{~dB} / \mathrm{km}$ loss and $16 \mathrm{ps} / \mathrm{nm} / \mathrm{km}$ dispersion coefficients showing; (a) $6,000 \mathrm{~km}$ reach and (b) as a function of reach at the optimum launch power. Solid lines;-high DMD 4-mode fibre (2.13ns/km), -low DMD 4-mode fibre $(0.17 \mathrm{~ns} / \mathrm{km})$, - high DMD 2-mode fibre, -low DMD 2-mode fibre, - standard SMF, - SMF with single channel $D B P,-S M F$ with 6 channel DBP, - SMF with 100 channel DBP (or OPC). Dotted lines; Optical regeneration with - no $O P C,-6$ channel $O P C,-100$ channel OPC.

Alternative technologies have also been proposed to enhance the capabilities of SMF, including DBP for optical super channels and optical regeneration. The capacity limits for a selection of configurations are shown by the remaining curves in Fig. 2a. In order to make comparisons with similar complexity, we consider two scenarios. Firstly, we consider the complexity is dominated by the opto-electronic components used to implement the system, and so we compare the performance DBP or optical regeneration of a super channel with 6 sub-channels to the performance of a few mode fibre system. The DBP results for 6 channels $(300 \mathrm{GHz}$ superchannels) show negligible improvement over single channel operation due to the influence of the large number of interfering channels over the full $5 \mathrm{THz}$ system bandwidth. Optical regeneration on the other hand (green and orange dotted lines) offer a substantial increase on capacity over conventional SMF, and an optical regenerator based SMF system (supporting PM-128QAM!) might be expected to outperform a 2-mode fibre. In the second scenario, we assume that the complexity is dominated by the digital signal processing. The complexity of a fewmode fibre system will scale with the maximum DMD and the number of ports, whilst that of a DBP system will scale with the number of steps per span which will itself be proportional to the ratio of dispersive to effective lengths, and so is proportional to the number of channels considered. Following these simple rules, we estimate that the DSP complexities for a 4-mode fibre, with low DMD if sufficient to allow DBP over the full C-band (red solid line). In this case, it is theoretically possible to compensate for all inter signal nonlinearities, such that the performance is limited by parametric amplification of amplified spontaneous emission, and the maximum capacity of a single SMF is comparable to that of a 2-mode fibre. If optical regeneration is included, then the SMF capacity approaches that of a four mode fibre. In this case, nonlinear compensation may be implemented using optical phase conjugation, although it is difficult to directly compare the complexity of an optical system incorporating C-band OPC and 100 channels of PM-1024QAM optical regeneration with a $12 \times 12$ MIMO system with a memory length of around 1 ns.

Figure $2 \mathrm{~b}$ illustrates that, with the exception of optically regenerated systems, the relative performance of each solutions is almost independent of transmission distance. This distance independent trend may be established from eq. (1), where it may be readily observed that for an inter channel nonlinearity dominated system, the optimum launch power is independent of the transmission distance and thus that SNR is inversely proportional to the number of spans. Consequently, conclusions relating to the relative merits of different fibre designs may be readily translated to different system reaches. In Fig. 3, we consider a simpler upgrade strategy for single mode fibres, optimisation of OSNR through amplifier placement, or the use of lossless Raman amplification. The optimum amplifier gain in order to minimise the sum of the amplifier output powers has recently been shown to be around $33 \mathrm{~km}$ for a $0.2 \mathrm{~dB} / \mathrm{km}$ loss coefficient. Figure 3 shows the SMF systems described above, plus systems employing lossless distributed Raman amplification (dashed lines) at this 
optimum amplifier spacing, alongside the few-mode fibre data for $100 \mathrm{~km}$ spacing. Such optimisation of the optical SNR clearly improves the performance of the SMF system (as it would the few-mode fibre system, but with increased mode coupling), eroding the margin between few-mode fibres and SMF fibres employing optimised electrical or optical signal processing to mitigate nonlinear effects.

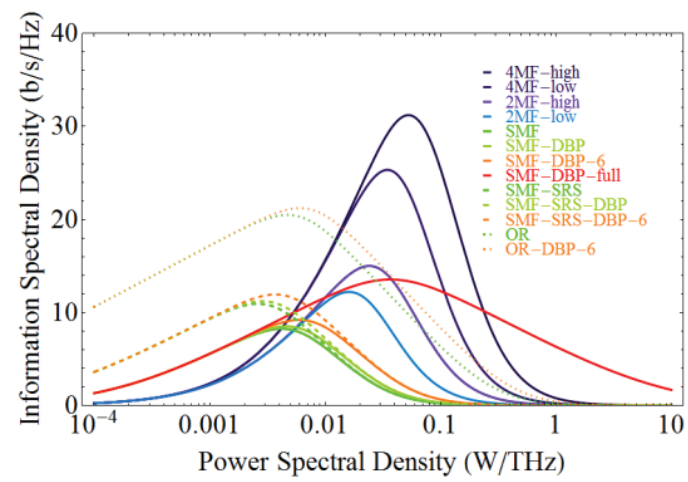

Figure 3. Information spectral density for few mode fibre systems with $100 \mathrm{~km}$ amplifier spacing and SMF with $33 \mathrm{~km}$ repeater spacing (solid lines), lossless distributed Raman amplification (dashed lines) and optical regeneration with $33 \mathrm{~km}$ regenerator spacing (dotted lines). Colours identify fibre configurations as per Fig. 2.

\section{CONCLUSIONS}

Capacity limit calculations applied to few-mode fibres indicate that the majority of the capacity increase associated with the additional modes would be recovered in an optimised system. However, this is clearly at the expense of increased signal processing complexity. Allowing similar levels of complexity for nonlinearity mitigation in a standard SMF allows substantial increase in the maximum capacity of these fibres, eroding the benefit of switching to few-mode fibres. Our results suggest that for point to point systems, an optimised SMF system with digital nonlinearity compensation could approach the maximum capacity of a 2 -mode fibre system, however, 4-mode fibre systems (and above) should offer appreciably larger capacities than a single SMF.

\section{ACKNOWLEDGEMENTS}

This work was partly funded by the European Communities 7th Framework Programme FP/2007-2013 grant 258033 (MODE-GAP), the EPSRC funded project UNLOC (grant number EP/J017582/1) and The Royal Society (Grant WM120035-TEST). The authors thank N. Mac Suibne and F.C. Garcia Gunning for assistance in the experimental confirmation of eq. (2).

\section{REFERENCES}

[1] P.P. Mitra, J.B. Stark: Nonlinear limits to the information capacity of optical fibre communications, Nature, 411, pp.1027-1030, 2001

[2] X. Chen, X, W. Shieh: Closed-form expressions for nonlinear transmission performance of densely spaced coherent optical OFDM systems. Optics Express, 18, 19039-19054 2010.

[3] A.D. Ellis: Current trends in optical communications, in Proc. ACOFT 2012, 2012.

[4] D. Qian et al:: 1.05 Pb/s transmission with 109b/s/ Hz spectral efficiency using hybrid single- and few-mode cores, in Proc. Frontiers in Optics, paper FW6C.3, 2012.

[5] A.D. Ellis, S. Sygletos: Phase sensitive regeneration based on SOAs, in Proc. OFC 2013, 2013

[6] T. Roethlingshoefer et al.: Multilevel amplitude and phase regeneration in a nonlinear amplifying loop mirror with a phase-sensitive amplifier, in Proc. ECOC 2012, paper Tu.1.A.3.

[7] M.D. Pelusi: All-optical pre-compensation of nonlinear fiber distortion for WDM $40 \mathrm{~Gb} / \mathrm{s}$ RZ-DPSK signals, in Proc. OECC 2012, pp. 901-902 (2012).

[8] D. Rafique, A.D. Ellis: Digital back-propagation for spectrally efficient WDM 112 Gbit/s PM m-ary QAM transmission, Optics Express, 19, pp. 5219-5224, 2011.

[9] Z. Tong et al.: Towards ultrasensitive optical links enabled by low-noise phase-sensitive amplifiers, Nature Photonics, 5, pp. 430-436, (2011).

[10] G. Rademacher et al:: Analytical description of cross modal nonlinear interaction in mode multiplexed multi-mode fibers, Photonics Technology Letters, 24, pp. 1929-1932, 2012.

[11] A.D. Ellis, S. Sygletos: Analytical calculations of the nonlinear Shannon limit for few mode fibers, submitted to ECOC 2013.

[12] S. Mumtaz, R-J. Essiambre, G.P. Agrawal: Nonlinear propagation in multimode and multicore fibers: Generalisation of the Manakov equations, Journal of Lightwave Technology, 31, pp. 398-406, 2012.

[13] D. Rafique and A. D. Ellis: Impact of signal-ASE four-wave mixing on the effectiveness of digital back-propagation in 112 Gb/s PM-QPSK systems, Optics Express, 19, pp. 3449, 2011.

[14] M.A. Sorokina, S.K. Turitsyn, Shannon capacity of nonlinear regenerative channels, available from arXiv: 1305.1537 (http://arxiv.org/abs/1305.1537), 2013. 